\title{
Towards Supporting the Existing Workplace Practices of a Community of Brazilian Healthcare Professionals
}

\author{
Roberto Calderon $^{1}$, Sidney Fels ${ }^{1}$, and Junia Anacleto ${ }^{2}$ \\ ${ }^{1}$ The University of British Columbia \\ ${ }^{2}$ Universidade Federal de São Carlos \\ roberto@alumni.ubc.ca, ssfels@ece.ubc.ca, \\ juniaedc.ufscar.bc
}

\begin{abstract}
With the increasing affordability of computers, displays and telecommunications, the scenario of introducing digital Information and Communication Technologies (ICT) into communities with little or no previous exposure to computing has become common place. Understanding how ICT affects the functioning of such communities is important for determining design and introduction strategies that can minimize the disruption of well established practices in said scenarios. We designed and introduced a ticketing system within a community of Brazilian healthcare professionals that have little or no previous exposure to computing. Visualizing individual people led to tasks directed towards particular individuals. Visualizing people interactions promoted open-ended and communal tasks. We observed that professionals circumvented the original design of the system to introduce unimplemented functionalities and support their well-established social-based information management practices.
\end{abstract}

Keywords: Visualization, Situated Displays, Healthcare, Collaboration, Social Capital.

\section{Introduction}

One of challenges regarding the introduction of Information Communication Technologies (ICT) into the workplace is understanding the effects of such technologies on the functioning of the communities that work there. In the present paper, we investigate the challenges of supporting the existing workplace practices within a community of healthcare professionals in a Brazilian centre for mental care. In particular, we aim at documenting how a community that has had little or no previous exposure to digital Information and Communication Technologies adopts the introduction of digital ICT and how the functioning of such community is affected by the new technology.

Through a previous ethnographic study [2] of this community, we uncovered a communication system based on verbal communication and pen-and-paper records 
that is sustained by informal socialization carried within the workplace throughout the day. Interpersonal communication and impromptu gatherings allow for efficient flow of information, that allows for flexible hierarchical structures and efficient task and patient status tracking.

Previous research has uncovered that situated public displays [10], and in particular the representation of ad-hoc work-flows $[5,13]$ can promote communication and enhance collaboration between healthcare professionals. However, the appropriation patterns and how such technology affects the social structure supporting collaboration within communities with low technical background is still unexplored. The present research focuses on determining the characteristics of ICT that could support the adhoc socialization practices that are essential to the community being studied.

Using a user-centred design we designed a ticketing system utilizing a situated public display and compared two types of visualizations: one depicting individual actions and a second one representing collaborative and group actions. We observed that the community of healthcare professionals modified the original purpose of the technology in order to accommodate unimplemented functionalities (e.g. capitalizing co-workers' names and adding task dates), and to fit their long-established information and communication practices (e.g. using the ticketing system to engage in conversation, and to publicize messages of sympathy and support towards co-workers).

\section{Related Work}

Systems to provide social awareness in order to promote collaboration within a workplace include the Notification Collage [6], and IM here [8]. These projects build upon a long history of research $[4,11]$ on the effect of public displays to promote collaboration within co-workers. Yet, this research often takes place within an office or research lab environment, which have different social dynamics and work-flow patterns from their healthcare counterparts. The benefits of public displays to promote collaboration in healthcare settings have been previously identified by Lasome et al. [10], who argue that public displays, like task boards, are naturally used to provide increased task management and shared awareness leading to more efficient problem solving. Within a healthcare setting, some researchers $[5,13]$ have investigated the use of task management systems to promote collaboration. This body of work suggests that the introduction of technology within the healthcare workplace changes how information is shared within members of the workplace, often modifying how interpersonal connections are made. Such investigations argue that the introduction of technology, particularly public displays to track and publicize workplace tasks, has many benefits such as an increase on collaboration [1] and socialization [9] patterns. The need to document the differences between HCI in the developed world and HCI for the developing one have been previously stressed in the literature [3]. Similarly, the need to investigate the role of ICTs for healthcare communities with little exposure to technology has been previously stressed in the literature [7]. 


\section{CAIS Hospital for Mental Care}

CAIS Clemente Ferreira (the hospital) located in Lins, São Paulo, Brazil, is a nonprofit state-run institution recognized as a Brazilian centre of excellence in mental health focusing on psychiatric disorders and neurological damages. The hospital currently employs over 380 professionals and provides care to more than 800 patients into eight care units serving different types of mental disorders and age groups. The architectural setting of the hospital - isolated urban location, corridors exceeding 100 meters in length and thick walls built in the early 1900s - make the installation of information and communication technologies a challenge, that paired with low governmental budgets have fostered a technology-free environment. In addition, many of the healthcare professionals have not been directly exposed to computing in their daily lives, as personal computers are rare and expensive in the area. As a result, healthcare professionals working at the hospital have developed, over the course of 25 years, an informational system based on pen-and-paper and verbal communication. The community studied is formed of auxiliary nurses, registered nurses, doctors, physiologists, phonologists and administration personnel. Following an ethnographic study we discovered that this community leverages socializing practices (e.g. storytelling) to communicate and collaborate.

\subsection{Social Work-Flows Currently Adopted by the Community}

In order to identify the natural practices adopted by the community being observed, we performed an ethnographic study where we shadowed members of the healthcare community [2]. In this study we identified the existence of informal gatherings and the use of storytelling as the core factors supporting collaboration between members of this community. Informal gatherings taking place on corridors, nursing rooms and hidden corners serve to promote verbal communication where tasks are informally delegated or tracked through playful conversations. This promotes an on going process of storytelling involving both personal and work-related stories that serve to efficiently track the status of important tasks, e.g. a new patient arrival. In this process of storytelling, professionals of all ranks are socially expected to participate and engage into vivid and friendly conversations. As a result, the otherwise inflexible job hierarchies and descriptions become flexible. That is, often people go off their job description and daily tasks to resolve issues discussed during these social meetings. We found that these naturally adopted collaboration practices are enhanced by a culturally rooted understanding of community where verbal and playful sociability is celebrated and exercised on every interpersonal encounter.

\section{Design of a Task Managing System to Support Informal Gatherings and Storytelling}

Our ethnographic study uncovered that daily tasks within the hospital are managed through informal gatherings and storytelling. In order to understand how ICTs can 
support these practices we designed a task managing application and compared two visualizations. A task managing kiosk, allowing healthcare professionals to manage tasks using a keyboard and mouse (Fig. 1), was designed following a user-centric iterative process. Two graphical representations of were made public for one month each, in order to measure the effects of different types of data visualization on the community. The first visualization (individual-centric) was designed to represent the individual count of resolved tasks. The second visualization (community-centric) was designed to represent individual actions in relationship to the community.

The first visualization (Fig. 2-A) consisted of circles representing each of the professionals of the wing. The radius of each circle was computed as the number of issues resolved by a person $R i$. The positioning of each circle was randomized at screen initialization. Understanding this visualization was straightforward, as people resolved more tasks, a circle with their name increased in size.

The second visualization (Fig. 2-B) focused on representing the relationship of each person to other members of the group and the group as a whole. Each person was represented as a circle whose radius was computed as the ratio of issues resolved by a person $R i$ to all issues resolved by all the members of the group $R$, i.e. $R i / R$. Circles were then arranged equidistantly from each other in a circle, and lines were drawn to represent links created between the person that created an issue and the person that resolved it. The width of such lines was computed as the ratio of issues resolved by a person $i$ and issued by person $j, R_{i \rightarrow j}$, to all issues resolved by person $i$, that is $\mathrm{R}_{\mathrm{i} \rightarrow j} / \mathrm{R}_{\mathrm{i}}$.

The size of circles represented people's ranking, rather than their number of tasks resolved. Therefore, changes in size would only be apparent if people solved considerably more tasks than their similarly-ranked peers. Additionally, thicker lines often meant that people would interact between each other more often. To make this clearer to the users, a legend was included on the bottom right part of the visualization explaining that larger circles "resolved more" and smaller circles "resolved less", while thinner lines depicted "less cooperation" and thicker lines represented "more cooperation".

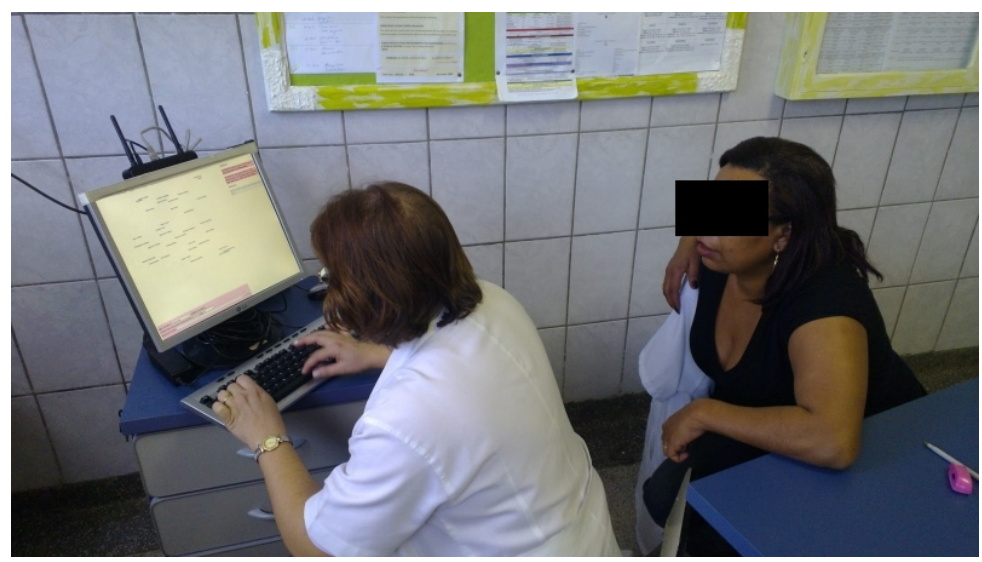

Fig. 1. Healthcare professionals use the public task-managing kiosk 


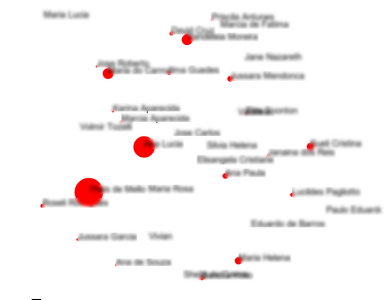

A

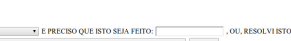

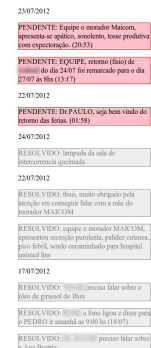
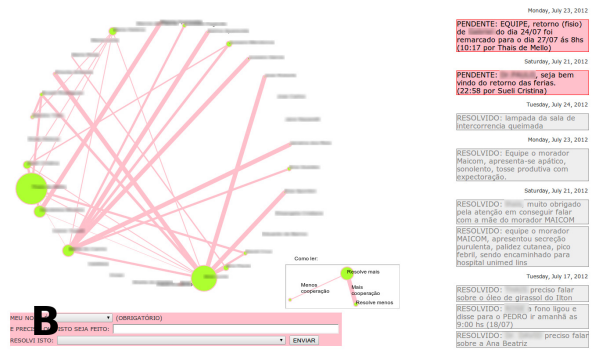

Fig. 2. Left: Individual-centric visualization showing people as isolated circles. (Screen-shot taken with all tasks collected during all 8 weeks.). Right: Community-centric visualization showing circles whose radius and connections depict people in relationship to the community or other people. (Screen-shot taken with all tasks collected during all 8 weeks.).

\section{$5 \quad$ Methodology Used for Semantic Analysis of Tasks}

In order to understand the motivations driving the usage of the kiosk we performed a semantic analysis of all tasks submitted through our prototype. Our analysis was theoretically grounded on the Interactive Places Framework proposed by Memarovic et al. [12]. The Interactive Places Framework proposes understanding content produced and consumed through public displays according to three strands: information production, means of disseminating such information and awareness of such information. Memarovic et al.'s conceptual framework models public display content as information created and consumed by either individuals or groups of people (i.e. with different levels of exclusivity) that when made available through the public display can be either explicit or implicit to a particular group of people or an individual (i.e. promoting different types of awareness).

\subsection{Exclusivity}

When content is directed towards anybody that comes in contact with the public display said content is said to be inclusive, however, when it is limited to a particular individual or group is said to be exclusive. When semantically analysing tasks submitted to our ticketing system we characterized exclusive content as tasks directed towards a particular healthcare professional or a group (e.g. "[MARTHA]: please fix the lamp on the bathroom" or "NIGHT TEAM: don't forget to stamp your forms after daily checkups."). We characterized inclusive content as generalized tasks not directed towards a particular person or a group of people, but to the whole community of healthcare professionals (e.g. "Patient $[\mathrm{X}]$ will not be receiving visitors this weekend.").

\subsection{Awareness}

Although a public display might be broadcasting information in a public manner not all people that come in contact with the content are able to make sense of it, or find it 
relevant to their interests and values. Explicit content portrays information about the interests and values of particular individuals or groups of people within the community. Implicit content is generalized information not pertaining a particular individual, or interest within the community. When semantically analysing tasks we considered explicit messages to be exemplified by calls for action from either particular individuals or the community (e.g. "[MARTHA]: please fix the lamp on the bathroom" or "TEAM don't forget to stamp your reports"). By contrast, tasks conveying generalized states of the workplace or unaddressed messages of conviviality were considered as implicit (e.g. "TEAM Have a good weekend" or "Emergency room light stopped working"').

\section{$6 \quad$ Findings}

We observed that professionals modified the original intended usage of the system to accommodate their existing practices. The original design of the ticketing system did not allow assigning tasks to particular individuals or groups. However, shortly after deployment some users begun addressing individuals by commencing new tasks with capitalized names (e.g. "[MARTHA] please fix...”). By the third week, this was considered a regular way to begin a message addressed to a particular individual or a group of people. Similarly, professionals created a way to determine task timings and deadlines by adding a date at the end of the submitted tasks. (e.g. "[MARTHA] attend Occupational Health day 15/7 8:30"). In both scenarios repetitive usage led to a common practice adopted by all members of the community of healthcare professionals.

We found that although the system was originally designed as a ticketing system to publicize tasks regarding the workplace healthcare professionals used it to post nontask messages to other professionals (e.g. "[Doctor X] welcome back from your vacation"), encouraging messages (e.g. "May we find strength to do our jobs today") and open ended socialization phrases (e.g. "Good day" or "Good weekend everyone"). In some cases we found traces of conversational posts (e.g. "I thank everyone for the support and help. May god bless you. Kisses." to which somebody replied "We are with you [Martha]" ).

\subsection{Semantic Analysis Findings}

We observed that during the individual-centric visualization the majority of tasks submitted were often explicitly directed towards a particular goal, person, or group of people (e.g. "Please stamp the examination request from [JACK]..." and "[MARTHA] please take care of my baby"). The majority of tasks submitted during this visualization often called for specific actions from members of the community.

By contrast, during the deployment of the community-centric visualization the number of implicit tasks depicting a status of the workplace (e.g. "[MARTHA], [GUS]'s wheel chair is wet") or messages to promote general awareness of a future or past event (e.g. "TEAM... [JACK] will go on the outing to the zoo day 04.12.2012...") greatly increased. 
We observed that during the individual-centric visualization the majority of task were exclusive, addressing particular healthcare professionals or groups of people, and often contained the capitalized name of the addressee in the body of the task. However, when the community-centric visualization was deployed the number of inclusive tasks, i.e. tasks not addressed to a particular individual and concerning anybody that came in contact with the display, surpassed that of exclusive, i.e. directed, tasks.

\section{$7 \quad$ Conclusion and Future Work}

The present study investigates how ICTs can help support the existing practices of a community of healthcare professionals in a Brazilian centre for mental care. We performed an ethnographic investigation of this community and uncovered that informal gatherings and storytelling are essential to the centre. These practices promote efficient task delegation and tracking, and a sense of community that has as benefit a better functioning workplace.

A ticketing system using a public kiosk was designed using a user-centred design process to support task tracking and two graphic representations of the data collected were compared. We found that a visualization depicting individual actions promoted tasks demanding specific actions from particular individuals or groups of people. By contrast, we found that a community-oriented visualization enticed healthcare professionals to publicize tasks that were open-ended and usable by all members of the community using the system.

Our future work aims at understanding the role that community reciprocity and common interests play in adopting digital information and communication technologies. We are applying the lessons learned in the above study to design a system to support open-ended and social notices that can be used by all the community.

Acknowledgements. We thank our funding agencies: GRAND, FAPESP, CAPES/DFAIT for their support. We thank the CAIS Hospital staff, MAGIC and our colleague, Jônatas Leite de Oliveira, for his help and insight with this project. The research was performed under the CAIS Ethical Statement.

\section{References}

1. Bardram, J.E., Hansen, T.R., Soegaard, M.: Awaremedia: a shared interactive display supporting social, temporal, and spatial awareness in surgery. In: Proceedings of the 2006 20th Anniversary Conference on Computer Supported Cooperative Work, CSCW 2006, pp. 109-118. ACM, New York (2006)

2. Calderon, R., Fels, S., Oliveira, J.L., Anacleto, J.: Understanding NUI-supported Nomadic Social Places in a Brazilian healthcare Facility. In: IHC 2012 (2012)

3. Chetty, M., Grinter, R.E.: HCI4D: hci challenges in the global south. In: CHI EA 2007, pp. 2327-2332. ACM, New York (2007) 
4. Fitzpatrick, G., Mansfield, T., Kaplan, S., Arnold, D., Phelps, T., Segall, B.: Augmenting the workaday world with elvin. In: Proceedings of the Sixth European Conference on Computer Supported Cooperative Work, pp. 431-450. Kluwer Academic Publishers, Norwell (1999)

5. Fouzi, L., Zidani, A., Chorfi, A.: A web application for supporting healthcare tasks with a groupware planning approach. In: 2012 International Conference on Information Technology and e-Services (ICITeS), pp. 1-6 (March 2012)

6. Greenberg, S., Rounding, M.: The notification collage: posting information to public and personal displays. In: Proceedings of the SIGCHI Conference on Human Factors in Computing Systems, CHI 2001, pp. 514-521. ACM, New York (2001)

7. Ho, M.R.: Communication and computing in health facilities of southwest Uganda. In: CHI EA 2010, pp. 4207-4212. ACM, New York (2010)

8. Huang, E.M., Russell, D.M., Sue, A.E.: Im here: public instant messaging on large, shared displays for workgroup interactions. In: Proceedings of the SIGCHI Conference on Human Factors in Computing Systems, CHI 2004, pp. 279-286. ACM, New York (2004)

9. Kim, J.-T., Lee, J.-H., Lee, H.-K., Paik, E.-H.: Design and implementation of the locationbased personalized social media service. In: ICIW 2010, May 9-15, pp. 116-121 (2010)

10. Lasome, C., Xiao, Y.: Large public display boards: a case study of an OR board and design implications. In: Proceedings of the AMIA Symposium (January 2001)

11. MacIntyre, B., Mynatt, E.D., Voida, S., Hansen, K.M., Tullio, J., Corso, G.M.: Support for multitasking and background awareness using interactive peripheral displays. In: Proceedings of the 14th Annual ACM Symposium on User Interface Software and Technology, UIST 2001, pp. 41-50. ACM, New York (2001)

12. Memarovic, N., Langheinrich, M., Alt, F.: The interacting places framework: conceptualizing public display applications that promote community interaction and place awareness. In: Proceedings of the 2012 International Symposium on Pervasive Displays, PerDis 2012, pp. 7:1-7:6. ACM, New York (2012)

13. Wilson, S., Galliers, J., Fone, J.: Not all sharing is equal: the impact of a large display on small group collaborative work. In: Proceedings of the 2006 20th Anniversary Conference on Computer Supported Cooperative Work, CSCW 2006, pp. 25-28. ACM, New York (2006) 